CLINICAL ETHICS

\title{
Ethics of HIV testing in general practice without informed consent: a case series
}

\section{J Fraser}

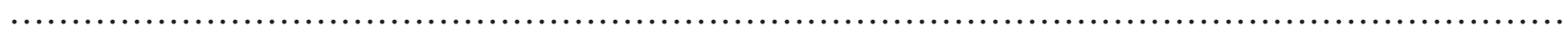

J Med Ethics 2005;31:698-702. doi: 10.1136/jme.2005.011734

This case series presents two general practice cases where HIV testing occurred, or results suggestive of HIV were received, before informed consent was obtained. Bioethical and professional principles are used to explore these dilemmas.

Correspondence to:

Dr John Fraser, Conjoint

Associate Professor,

Discipline of General

Practice, University of

Newcastle, Callaghan

2308, Australia; john.fra-

ser@hnehealth.nsw.gov.au

Received 19 January 2005 Accepted for publication 23 March 2005
C ase 1: A 40 year old man presented in 1990 for a life insurance medical and routine blood tests including HIV antibodies. The HIV test was refused. Despite prompting, the reasons for this were not volunteered. Several days later, I received the patient's results. This included an HIV antibody test identifying the patient. This was in contravention of our practice policy which is to code HIV results. The pathology request form was reviewed. This showed an HIV test had been added in different handwriting. Practice and pathology staff could not explain the source of this writing. The patient returned for his results. Fortunately, the patient raised the issue of the HIV test himself. He had changed his mind and had himself written on form to the pathologist. The implications of his negative result were explained after discussing the patient's risk factors for HIV with him.

Case 2: A 43 year old married clergyman presented in 2000 with a recurrent infection of his little toe. This developed into a pyodermic granuloma and was treated by curretage and diathermy. The preliminary pathology report queried Kaposi's sarcoma and further staining was requested. The patient failed to call for his results. Based on my knowledge of this patient and his family, there were no risk factors for HIV infection. I considered whether to arrange an interview with this patient as soon as possible in order to take a detailed sexual history with a view to urgent HIV testing. I felt this course of action was likely to cause distress, that the risk of HIV in this patient was low, and that the delay in getting a final histological diagnosis was unlikely to alter the clinical course. The subsequent pathology report confirmed granulation tissue only.

\section{DISCUSSION}

General practitioners (GPs) owe duties to their patients to detect HIV infection and to offer pretest counselling before testing for serious communicable diseases. ${ }^{1}$ Patients with mental illness, who are critically ill or unconscious may lack the capacity to give informed consent. ${ }^{1}$ In these circumstances, researchers ${ }^{2}$ and clinicians ${ }^{3}$ the request form before delivering the request have justified HIV testing without consent. These arguments are rejected by others: some contend that failing to seek a patient's consent before HIV testing is always wrong ${ }^{4}$; while others suggest testing should only occur when it will alter the clinical care of the patient. ${ }^{6}$ The General Medial Council specifies rare circumstances when HIV testing may occur without consent. ${ }^{7}$ These guidelines emphasise the need to discuss actions with a colleague in order to ensure that one's actions are reasonable and professional. Despite this literature, there is limited discussion concerning potential lapses in these procedures which can arise in general practice.

Patient autonomy and self determination underpin informed consent before HIV testing. ${ }^{1}$ These cases illustrate times when informed consent may not be obtained in general practice: either because of systematic errors in the process of obtaining informed consent, coding, and receiving results, or because other pathology tests, such as skin biopsies, can act as surrogate markers of HIV.

In responding to these situations, GPs need to consider the organisational and legal dimensions of practice and their professional and ethical roles. These aspects of practice are central domains of general practice. ${ }^{8}$

Inadvertent HIV testing without consent raises two issues: firstly, the patient has the right to know what has happened and to be asked whether they wish to know their results; and secondly, the consult is more difficult to predict and plan, as the patient's response to a positive or negative result has not been explored. In my opinion, more harm may be done to the doctor/ patient relationship by deliberately concealing knowledge of the result, even if it is negative. The patient may find out at a later date of testing, if they receive treatment from a colleague or a locum of the practice and are asked about the reasons for the recent HIV test. This would be worse than discussing the error at the time it was discovered.

General practitioners have legislated guidelines concerning pretest counselling which can be considered as minimal standards of care. ${ }^{17}$ The importance of documenting pretest counselling or a patient's refusal to testing is illustrated by case 1 where HIV testing occurred because of a systems error. Documentation needs to cover what was discussed and the patient's capacity to consent to, or decline, HIV testing. ${ }^{17}$ I reflected on this incident, and have since changed my clinical practice: I now always take a coded HIV venous sample myself. Using this system, I can better ensure the confidentiality of my patients, 
and reduce the risks of patients organising an HIV test without pretest counselling. If a positive result had occurred in case 1, the GP's professional and ethical role would have become more complex. The duty of care would expand to cover both the patient and their partner. ${ }^{9}$ Failure to notify the partner of the patient's HIV status can represent a breach in duty of care. ${ }^{179}$

Kaposi's sarcoma can occur spontaneously in the elderly, however, in younger men it is usually a sign of AIDS. ${ }^{1}$ General practitioners need to be very alert to the possibility of HIV infection. Acute HIV seroconversion and HIV infection can mimic conditions commonly seen in general practice including lymphadenopathy, fever, myalgia, cough, diarrhoea, candidiasis, and seborrhoeic dermatitis. Incorporating sexual and drug histories into routine clinical care can identify patients at increased risk of HIV. Case 2 demonstrates that the general practitioner needs to consider the possibility of HIV in association with the patient's risk factors. Suggesting a possibility of HIV where it is unexpected and the risk is low, can in itself cause anxiety and distress. A valid argument to defer raising the possibility of an HIV diagnosis was made and supported by the subsequent histology in case 2 .

This case series demonstrates bioethical dilemmas which can arise in HIV medicine in general practice. The GP plays an important role in assessing patient risks factors, facilitating early detection of HIV, and ensuring that testing occurs with informed consent. At times, these duties can compete with each other and GPS need to balance the rights of their patients and partners to optimise care.

\section{REFERENCES}

1 Australasian Society for HIV Medicine. HIV/viral hepatitis: a guide for primary care. Canberra: National Capital Printing, 2001.

2 Bhagwanjee D, Muckart D, Jeena $P$, et al. Does HIV status influence the outcome of patients admitted to a surgical intensive care unit? A prospective double blind study. BMJ 1997;314:1077-81.

3 Newton P, Newsholme W, Brink N, et al. Acute meningoencephalitis and meningitis due to primary HIV infection. BMJ 2002;325:1225-6.

4 Kale R. Commentary: Failing to seek patients' consent to research is always wrong. BMJ 1997;314:1081-2.

5 Malin A, Lockwood D. Informed consent: study in which patients had HIV tests could be designed differently. BMJ 1997;315:251-2.

6 Sommerville A. Commentary: Is testing for HIV without consent justifiable? BMJ 2002;325:1226-7.

7 General Medical Council. Serious communicable diseases. London: General Medical Council, 1997.

8 Royal Australian College of General Practitioners. Training program curriculum [2nd ed]. Melbourne: Royal Australian College of General Practitioners, 1999

9 Hirsch D. Doctor's duty of care to a patient's sexual partners. Australian Health Law Bulletin 1999;8:53-6.

\section{HIV testing and informed consent}

\section{Frith}

\section{People should be allowed to decide how and where they wish to be tested for HIV without there being a formal requirement for pretest counselling}

n his paper, Ethics of HIV testing in general practice without informed consent, Fraser argues that pretest counselling and informed consent are pillars of the ethical conduct of HIV testing. In my response I want to look critically at these contentions. While I will agree with Fraser that it is always necessary to get informed consent from a patient for an HIV test I will argue that an emphasis on pretest counselling as a prerequisite for testing can actually undermine a patient's autonomy, the very principle that informed consent seeks to promote.

This response will start with an analysis of Case Two as this highlights the fundamental importance of informed consent. It will then go on to look at Case One and show how the special application of informed consent in the field of HIV testing can, in certain circumstances, lead to a reduction in patient autonomy.

\section{CASE TWO}

In Fraser's second case a 43 year old married clergyman presented with a recurrent infection of his little toe and the preliminary lab reports queried Kaposi's sarcoma. The general practitioner (GP) thought that because of the patient's lifestyle there was little risk of HIV infection, "suggesting the possibility of HIV, when it is unexpected and the risk low, can in itself cause anxiety and distress". On these grounds the GP did not call the patient in for an HIV test.

Some might argue that the GP should have tested the clergyman. This could have been done in one of two ways: by seeking his explicit consent for an HIV test, or by getting blanket consent for a range of tests, including HIV, without HIV being explicitly mentioned.

The first option, while possibly causing the patient some distress, would, had HIV been confirmed, have been seen by many to be the correct course of action. There are clearly important benefits, which would outweigh the anxiety caused by the test itself, to both the patient and his immediate contacts to knowing his positive HIV status. In this situation a risk assessment was made that it was unlikely the clergyman would be HIV positive, because of his lifestyle, and this proved to be correct.

Some practitioners, however, would argue that in instances such as Case Two a more effective course of action would have been to simply get blanket consent for an unspecified range of tests that would have included HIV.

This argument has been put forward in a recent $B M J$ editorial. According to this editorial it is time to, "lower the threshold for HIV testing", enabling doctors, in certain circumstances, to test for HIV without obtaining a specific consent for the HIV test. "We propose that if a patient freely consents to be investigated, a doctor can initiate tests aimed at excluding serious diseases without an in depth discussion of all possible results, provided the test result, positive or negative, should benefit the patient ${ }^{\prime \prime} .{ }^{1}$ To support their case Manavi and Welsby make an analogy with the case of tuberculosis testing. Often patients do not explicitly consent to the taking of a sputum sample for acid fast bacillus to exclude or confirm tuberculosis, as it is seen as just one of many tests performed to make an accurate diagnosis. They infer from this that HIV should be treated in a similar fashion and be included in a range of non-specified diagnostic tests to which a patient would give blanket consent.

Arguments in favour of such an approach to HIV testing are broadly consequentialist in nature. ${ }^{2}$ It would improve the diagnostic capabilities of doctors if they could, on suspecting HIV infection, simply include it in a battery of tests without having to obtain specific consent. As demonstrated by Case Two, 
doctors are wary of suggesting the possibility of HIV to patients and such a reluctance to test may result in a delayed diagnosis and compromised care for the patient. ${ }^{3}$ It could also be argued that this blanket consent to HIV testing would reduce the "unnecessary" distress of patients - that is, if the test eliminated HIV-and increase the levels of HIV testing in general medicine-a desirable outcome.

Such blanket testing, although arguably having some benefits, clearly goes against current notions of good practice. Although there may be good arguments to suggest that HIV should be treated like any other disease it is another matter to suggest that HIV testing should embrace what is increasingly seen as bad practice in other areas. Current GMC guidelines state: "You must obtain consent from patients before testing for a serious communicable disease ${ }^{\prime \prime}{ }^{4}$

Although the GP in Case Two did not go ahead with an HIV test the principle of informed consent was at the centre of his decision and he acted in accordance with current guidelines. Had the GP decided to test, wishing to uphold the principle of informed consent, he would have been obliged to tell the patient about the HIV test. It was the possible anxiety to the patient created by raising the possibility of a test that was felt to outweigh the risks of not testing.

I want to show how the special practices that have grown up around obtaining informed consent for HIV testing can, paradoxically, sometimes have the effect of undermining a patient's autonomy-the very principle which underpins informed consent. It is to this specific problem, exemplified in Fraser's first case, to which I will turn.

\section{CASE ONE}

In Fraser's first case a patient refused consent for an HIV test when requesting life insurance. Subsequently, the patient authorised a test on the request form without the GP's knowledge. For Fraser, the ethical difficulty presented by this case was that the minimal standard of care, providing pretest counselling, was not given and after reflecting on this incident he changed his practice to, "reduce the risk of patient initiated HIV testing".

Inherent in Fraser seeing "patient initiated testing" as morally problematic is the view that HIV is somehow different from other diseases and therefore "special" ethical considerations pertain to the conduct of testing. ${ }^{5}$ The major factor that has often distinguished HIV testing from other medical tests is that people have had to have counselling as a prerequisite for having a test. Whereas with other diseases counselling might be offered or made available if requested, with HIV a patient has had to undergo counselling in order to have a test. This is one aspect of what has been called "HIV/AIDS exceptionalism"-treating HIV differently from other diseases. ${ }^{67}$

The question we have to ask is should HIV be treated differently. Should there always be, as Fraser suggests, obligatory pretest counselling or should HIV be treated the same as other conditions of a similar severity?

Fraser says the reason for pretest counselling for an HIV test is to promote and safeguard patient autonomy. I would argue that such counselling may promote autonomy in one way, in that it provides information to patients so they can make a fully informed decision. However, it can undermine patient autonomy in another way, by forcing patients to receive face to face counselling, which, given the nature of how the disease is transmitted, they may not want to have. ${ }^{8}$ An insistence on pretest counselling can be seen as a paternalistic response that actually reduces, rather than fosters, a person's autonomy. Thus, I would disagree with Fraser and argue that making pretest counselling a requirement for having an HIV test should no longer be seen as an ethical necessity. This is not to say that if people want counselling it should not be available but, that if they do not, their decision should be respected.

A further element of Case One that Fraser sees as morally problematic is that the patient had initiated the test himself. On this basis, Fraser would be against home or self testing for HIV. Such opposition clearly raises important ethical considerations. The recent trend in health care, stimulated by an emphasis on patient autonomy, has been for greater self diagnosis and screening. As it has become more widely recognised that patients can make informed decisions without the direct intervention of healthcare professionals, individuals have been encouraged to take responsibility for their own health. Self breast examination and pregnancy testing are all routinely carried out at home and there is-for example, a self test available for occult blood in stools, to screen for colorectal cancer.

If it is accepted that people should be allowed to make informed decisions as to how they are to be tested then there is clearly a very strong ethical case for saying that patients should be able to self or home test for HIV. This is already the case in the USA where a Federal Drug Agency (FDA) approved home test has been available since $1996 .{ }^{8}{ }^{9}$ While recognising that there are many issues raised by self or home tests, ${ }^{10}$ I would argue, nevertheless, that where the possibility of accurate testing exists we should allow people to choose where and how they have an HIV test. It is possible that a substantial number of people would prefer to conduct the test at home. ${ }^{810}$ As an editorial in the Canadian Medical Association Journal says: "Where the technology exists, why should the public not have autonomy and privacy in obtaining important health information [on their HIV status]?"11

\section{CONCLUSION}

Fraser's cases raise some interesting dilemmas faced by practitioners over the conduct of HIV testing. I have agreed with Fraser that it is always necessary to get informed consent from a patient for an HIV test and, indeed, this is now part of standard medical practice for any serious disease. However, his support for obligatory pretest counselling as a means of obtaining informed consent can be questioned. I have argued that such a requirement can, paradoxically, sometimes have the effect of undermining a patient's autonomy-the very principle that informed consent seeks to promote. Hence, I would support the removal of the requirement for pretest counselling, something that is already happening in the UK, and let people decide how and where they wish to be tested for HIV.

doi: 10.1136/jme.2005.012328

Correspondence to: Lucy Frith, Lecturer in Health Care Ethics, Department of Primary Care, The University of Liverpool, The Whelan Building, Brownlow Hill, Liverpool, L69 3GB, UK; I.j.frith@liverpool.ac.uk

Received 28 June 2005

Accepted for publication 29 June 2005

\section{REFERENCES}

1 Manavi K, Welsby P. HIV Testing should no longer be accorded special status. BMJ 2005;330:492-3.

2 Parker M. Consent to HIV testing and consequentialism in healthcare ethics. HEC Forum 2004; 16:45-52.

3 Newton P, Newsholme N, Brink N, et al. Acute meningoencephalitis and meningitis due to primary HIV infection. BMJ 2002;325:1225-6.

4 General Medical Council. Serious communicable diseases. London: General Medical Council, 1997.

5 Pinching A, Higgs R, Boyd K. The impact of AIDS on medical ethics. JME 2000;26:3-8

6 DeCock K, Johnson A. From exceptionalism to normalisation: a reappraisal of attitudes and practice surrounding HIV testing. BMJ 1998;316:290-3.

7 Rosenbrock R, Dubois-Arber F, Moers M, et al. The normalization of AIDS in western European countries. Soc Sci Med 2000;50:1607-29.

8 Merson H, Feldman E, Bayer R, et al. Rapid self testing for HIV infection. Lancet 1997;349:352-3.

9 www.fda.gov/cber/products/testkits. htm (accessed 1 May 2005).

10 Speilberg F, Levine R, Weaver M. Self testing for HIV: a new option for HIV prevention? Lancet Infect Dis 2004;4:640-6.

11 Anon. Home HIV testing: why not in Canada? CMAJ 2000;162:1545. 
"sensitive" tests, as is already done in some areas of the United Kingdom.

Although case 1 exposes a systemic

\section{Commentary on Ethics of HIV testing in general practice without informed consent: a case series}

\section{K Sokol}

\section{Case 1 reminds us that patients have duties too, while case 2 presents an instance of justified withholding of information}

$\mathrm{H}$ ow refreshing to read these two cases! No conjoined twins, fantastical chimeras, or other incredible scenarios at the fringes of medical reality. Each case highlights the practical and theoretical difficulties that doctors face in their everyday practice.

Case 1: In case 1, the patient, who had declined an HIV test, changed his mind and requested an HIV test on the request form without informing the doctor. The pathologist performed the tests and returned the results to the unsuspecting doctor, who duly investigated the matter. The doctor's anxiety at the situation, as well as the worries of the entire practice and pathology laboratory, must have been deeply unpleasant. Thus the patient, no doubt unintentionally, caused much distress by requesting the HIV test. He was, at the very least, guilty of a lack of foresight and consideration. As a minimum, he should have informed the doctor of his last minute alteration. This would have enabled the doctor to discuss the implications of the test, to organise appropriate counselling, and, most importantly, to receive the results in full knowledge of the situation.

As Dr Fraser points out, the experience of case 1 reinforces the importance of taking good medical notes. Even if the patient's refusal had been documented in the notes, however, this would not have prevented him from requesting the HIV test, and indeed other tests, on the blood form. Pharmacists may still occasionally be faced with situations when they are unsure whether the doctor prescribed 10 capsules of a drug, or 110 capsules, as the patient may have added a " 1 " in front of the doctor's original prescription. Similarly, how can doctors and pathologists guarantee that patients will not modify the form as soon as they leave the surgery?

Whatever their immediate consequences, mishaps in medicine often provide opportunities to improve practice. Once the causal factors are identified, changes can be made and implemented. So what can be done to prevent reoccurrences of incidents such as those in case 1 ?

First, patients can be taught to refrain from ordering tests without prior approval by their doctor. The doctor/ patient relationship, like all relationships, is not one sided. Patients have duties too. In general, they should be truthful, cooperative, courteous, considerate, and trustworthy. All of these duties should have compelled the patient in case 1 to inform the doctor of his alteration. Whether-and, if so, how-to instil these duties in the doubtless small number of patients who are unaware of them is beyond the scope of this brief commentary. None the less, whatever the efforts to raise awareness of patients' duties, it would be unrealistic to expect all patients to abide by them. Our solution should operate successfully in a non-ideal world where some patients are, as one general practitioner put it, "a pain".

The key concern arising from the case relates to the content of pathology request forms. If in possession of a written form, it may be easy for patients to order additional tests without the doctor's knowledge. Doctors trust patients to deliver the request forms and specimens from their office to the next step of the delivery process without tampering with them. To reduce the likelihood of tampering and to increase detection if it does occur, computer generated forms may be preferable to handwriting. Delivery of forms and specimens without involvement of the patient would also reduce the possibility of unauthorised changes. Whether such changes are practically desirable, given the time constraints and the rarity of abuse, is an issue for consideration. If pathologists suspect a discrepancy in authorship-for example, because of different handwriting-they should contact the general practitioner for confirmation. Alternatively, separate forms could be used for HIV or other weakness in the completion and transit of pathology request forms, its main interest lies in the important but neglected questions it raises about the role and duties of patients.

Case 2: Case 2 illuminates a common dilemma for doctors: when should a possible but very uncertain diagnosis be communicated to a patient?

The doctor had two opportunities to convey the possibility of HIV. The first was when he investigated the granuloma. Because there is a link between pyodermic granuloma and Kaposi's sarcoma (KS) and, in turn, between KS and HIV, the doctor could have informed the patient of this unlikely possibility. Now, it is probable that $\mathrm{Dr}$ Fraser did not even suspect KS at this point, let alone HIV. The probabilities of each, in light of the clinical evidence and the doctor's knowledge of the patient, would have seemed minuscule.

Although one often reads of "fully informed consent" in the medical literature, such a state of total knowledge and understanding is a myth. It is impossible for doctors to disclose all the risks and possible side effects of a drug, treatment, or procedure. Not only would attempting to do this impossible task be practically disastrous, prolonging waiting times to unacceptable levels, but it would be medically and psychologically undesirable. A headache is very rarely a brain tumour, and there seems little point in alarming all those with headaches of this grim possibility. Only when the likelihood reaches a certain threshold of significance should the suspicion be communicated. By anyone's account, the threshold for disclosing the possibility of HIV was surely not reached at the time of investigating the pyodermic granuloma. Even if Dr Fraser had entertained the possibility, it would arguably have been inappropriate to reveal this thought for fear of unduly distressing the patient.

The second opportunity arose following the suspected KS. A few points should be considered. Firstly, the pathology report only queried KS. The diagnosis was thus far from positive. Secondly, there is no necessary link between KS and HIV. Kaposi's sarcoma may occur independently of HIV. Thirdly, the gravity of KS varies considerably from relatively harmless lesions to fairly rapid death-as in African KS-depending on the type of $\mathrm{KS}$, the extent of organ involvement, and various patient characteristics. ${ }^{1}$

Dr Fraser's decision to withhold the suspicion was based on three reasons: 
1. Discussing the issue of HIV would very probably cause distress.

2. The probability of HIV was low, hence discussion of this possibility would be unnecessary.

3. The clinical course would not be altered by the delay to obtain confirmation of KS.

Of these, only the second reason is valid. The fact that a disclosure is likely to distress a patient is not, in itself, a sufficient reason to withhold information. Unless the anguish caused by the revelation would be extremely harmful, the crucial importance of information for autonomous decision making surely outweighs the benefits of non-disclosure. Furthermore, it is difficult to know how others will respond to bad news and, even when it is highly distressing, the deleterious effect of adverse disclosures is often temporary. In cases of sexually transmitted diseases, the communicable nature of the disease provides additional weight to the prima facie duty to disclose.

Although a delay may not alter the clinical progression of the disease in the individual patient, HIV can be transmitted to others. Informing patients of this possibility can help reduce the likelihood of transmission by allowing them to control their behaviour. For this reason, suspicions of communicable diseases should be shared with the patient at a lower threshold of certainty than non-communicable conditions.

Without detailed knowledge of the patient, his history, and his lifestyle, and the probability of the initial diagnosis of $\mathrm{KS}$, I can only come to a tentative decision about what I would have done myself in Dr Fraser's situation, but, based on the information provided in the case description, I am inclined to support his conclusion. In light of the queried test results, the probabilistic link between KS and HIV, and the patient's "risk free" lifestyle, the probability of HIV probably fell below the threshold of likelihood requiring disclosure, even for a condition as serious and transmittable as HIV. One caveat, however, about the dangers of assuming the absence of risk factors: even a prolonged friendship with a patient may not reveal an unavowed penchant for drugs or hazardous sexual behaviour. A good doctor should never rule out these possibilities, however unlikely they may appear.

William Osler called medicine "an art which consists largely in balancing probabilities". ${ }^{2}$ This case is a wonderful illustration of this.

\section{ACKNOWLEDGEMENTS}

Many thanks to Samantha Hettige, Raanan Gillon, Andy Chivers, and Frances Carter for their invaluable assistance.

doi: 10.1136/jme.2005.012344

Correspondence to: Daniel K Sokol, Medical Ethics Unit, Department of Primary Health Care and General Practice, Imperial College London, Reynolds Building, St Dunstan's Road, London W6 8RP, UK; daniel.sokol@talk21.com

Received 26 March 2005

Accepted for publication 20 April 2005

\section{REFERENCES}

1 American Cancer Society. Detailed guide: Kaposi's sarcoma. www.cancer.org/docroot/ cri/content/cri_2_4_1x_what_is_kaposis sarcoma_21.asp? sitearea $=$ cri (accessed 24 Jun 2005).

2 Osler W. Aphorisms from his bedside teachings and writings. New York: Henry Schuman Inc, 1950 